FORMATION Formation emploi

Revue française de sciences sociales

139 | Juillet-Septembre 2017

De l'autonomie dans les parcours professionnels

\title{
Les chômeurs âgés face au « vieillissement actif ». Une comparaison France - Royaume-Uni
}

older workers and active ageing. A comparison between France and the UnitedKingdom

Ältere Arbeitssuchende und „aktives Altern“. Ein Vergleich zwischen Frankreich und dem Vereinigten Königreich

Los desocupados mayores frente al « envejecimiento activo ». Una comparación Francia-Reino Unido

\section{Claire Lefrançois}

\section{CpenEdition} Journals

Édition électronique

URL : http://journals.openedition.org/formationemploi/5126

DOI : 10.4000/formationemploi.5126

ISSN : 2107-0946

Éditeur

La Documentation française

Édition imprimée

Date de publication : 15 octobre 2017

Pagination : 15-32

ISSN : 0759-6340

Référence électronique

Claire Lefrançois, «Les chômeurs âgés face au « vieillissement actif ». Une comparaison France Royaume-Uni », Formation emploi [En ligne], 139 | Juillet-Septembre 2017, mis en ligne le 15 octobre 2019, consulté le 30 octobre 2020. URL : http://journals.openedition.org/formationemploi/5126 ; DOI : https://doi.org/10.4000/formationemploi.5126 


\title{
Les chômeurs âgés face au « vieillissement actif». Une comparaison France - Royaume-Uni
}

\author{
Claire Lefrançois \\ Maître de conférences en sociologie, UMR CITERES (Cités, Territoires, Environnement et \\ Sociétés), université de Tours
}

Résumé

\begin{abstract}
Les chômeurs âgés face au « vieillissement actif ». Une comparaison FranceRoyaume Uni

Le vieillissement actif, promu depuis la fin des années 1990 par les instances européennes, conduit à inciter les individus à rester actifs le plus longtemps possible. Nous examinons la manière dont ce référentiel se diffuse dans deux contextes nationaux, le Royaume-Uni et la France. À partir de l'analyse d'une cinquantaine d'entretiens menés auprès de chômeurs, nous montrons que ce référentiel ne trouve pas le même écho selon les pays et les milieux sociaux.

Mots clés : travailleur âgé ; chômeur; chômage ; durée d'activité; politique de l'emploi ; politique sociale ; indemnisation du chômage ; retraite ; cheminement professionnel ; psychosociologie du travail ; comparaison internationale ; France ; Royaume Uni
\end{abstract}

Abstract

Older workers and active ageing. A comparison between France and the United-Kingdom

Promoted since the end of the 1990s, active ageing encourages people to stay active as long as possible. We examine how this policy circulates in the United Kingdom and France. Based on the analysis of fifty interviews conducted with unemployed older workers, we show how it spread depending on countries and social relations.

Keywords: Elderly worker; unemployed person; unemployment; duration of activity; employment policy; social policy; unemployment benefit; retirement; occupational paths; occupational psychology; international comparison; France; United Kingdom

Journal of Economic Literature: J 24 ; J 26

Traduction : Auteure 
À la fin des années 1990, le concept de "vieillissement actif» émerge sur la scène internationale et se transforme peu à peu en référentiel de l'action publiquel (Viriot Durandal, Moulaert, 2014). En Europe, le vieillissement actif est surtout perçu sous l'angle de l'emploi. La Commission européenne s'est emparée de ce référentiel dans l'objectif de prolonger les carrières professionnelles, en considérant que le vieillissement actif consiste à travailler plus longtemps (Jolivet, 2002)2.

Dans un contexte d'inquiétudes sur le financement des retraites, ce référentiel offre en effet un argument pour inciter les plus âgés à travailler le plus longtemps possible afin, selon les pouvoirs publics, qu'ils demeurent des sujets autonomes indépendants des systèmes d'assistance (Biggs, 2001 ; Duncan, 2008). En ce sens, il s'inscrit en cohérence avec les politiques d'activation qui visent à ce que les prestations sociales, comme les allocations chômage, soient davantage conditionnées à l'activité sur le marché du travail (Barbier, 2002).

Avec la Stratégie européenne pour l'emploi, l'Union européenne incite ainsi les États membres à se saisir du vieillissement actif afin de prolonger les fins de carrière et de renforcer la place des plus âgés sur le marché du travail. La prise en compte des carrières, c'està-dire de manière plus analytique, des parcours professionnels et de leur structuration, est donc une préoccupation des politiques publiques. Les États européens ont alors entamé des réformes des retraites et ont introduit des mesures destinées à favoriser l'emploi en fin de carrière (Guillemard, 2010). À la fin des années 1990, au Royaume-Uni, et à partir du milieu des années 2000, en France, l'emploi des actifs vieillissants a été mis à l'agenda.

Dans cet article, nous proposons d'analyser la manière dont ce référentiel se décline, en France et au Royaume-Uni, deux pays caractérisés par leurs divergences en termes de politiques de l'emploi et de systèmes de protection sociale, en particulier en matière d'activation qui prend des visages contrastés selon les pays (Barbier, op. cit.).

Dans quelle mesure le vieillissement actif fait-il sens dans les deux pays étudiés ? Y trouvet-il un écho favorable? Rencontre-t-il les directions prises par les politiques publiques ? Qu'en est-il des aspirations en matière d'emploi des individus, notamment des chômeurs en fin de carrière? Ceux-ci constituent un public ad hoc pour approcher les effets des politiques publiques qui s'inscrivent dans le référentiel du vieillissement actif. En effet, cette catégorie est d'abord perçue à travers son éloignement par rapport à l'emploi et est la plus discriminée en raison de son âge. Quand la question de son retour à l'emploi est mise à l'agenda, la littérature statistique et sociologique pointe le risque important de connaître

1. La notion de référentiel renvoie à des normes et des valeurs qui produisent des cadres d'interprétation et qui, ce faisant, "donnent du sens à un programme d'action publique en définissant des critères de choix et des modes de désignation des objectifs" (Muller, 2010, p. 556).

2. En 1999, la Commission envisageait le vieillissement actif dans une approche large sur les cycles de vie, sans se centrer exclusivement sur l'emploi, mais avec l'adoption de la Stratégie européenne pour l'Emploi en 2002, il est uniquement envisagé dans la perspective de la prolongation des carrières. Pour sa genèse, nous nous permettons de renvoyer à : Jolivet, op. cit. ; Viriot Durandal, Moulaert, op. cit. . 
un chômage de longue durée et les chances relativement tenues de réemploi en raison de sa proximité avec la retraite (Macnicol, 2006 ; Guillemard, op. cit.). À l'heure où les politiques publiques allongent la durée des carrières professionnelles, comment ce public envisage-t-il le retour à l'emploi ? Appréhender la diffusion du vieillissement actif à partir des publics visés par l'action publique, notamment ceux perçus comme les plus éloignés de l'emploi, permet d'étudier la réception du vieillissement actif, d'appréhender ses effets et ses usages.

\section{Encadré 1 : Méthodologie}

Cet article est issu d'une thèse de sociologie étudiant les politiques de l'emploi visant les chômeurs âgés. II s'appuie sur l'analyse d'une cinquantaine d'entretiens réalisés entre 2009 et 2010, auprès de chômeurs anglais (19) et français (28). Ces entretiens, dont la durée varie de quarante-cinq minutes à une heure et demie, traitent de l'expérience du chômage, du parcours professionnel antérieur et du rapport à la retraite.

Nous avons rencontré ces chômeurs via des ateliers de recherche d'emploi dédiés aux plus de 50 ans. Ce critère d'âge est utilisé de manière souple en France, où il fait office de point de repère ; en outre, des chômeurs de moins de 50 ans peuvent y participer. C'est ainsi que nous avons pu interviewer des quadragénaires. En revanche, nous n'y avons pas rencontré de personnes de plus de 60 ans (ce qui s'explique par un âge légal de la retraite plus bas qu'au Royaume-Uni, une culture de la sortie précoce encore prégnante et des dispositifs leur permettant de quitter le marché du travail avant l'âge de la retraite).

Au Royaume-Uni, en revanche, seuls les plus 50 ans peuvent fréquenter ces ateliers. En outre, nous y avons croisé régulièrement des sexagénaires en raison d'un âge légal de la retraite plus élevé, conjugué à l'absence de dispositifs permettant de quitter le marché du travail précocement. C'est la raison pour laquelle nous avons rencontré des chômeurs plus âgés (la moyenne d'âge des chômeurs anglais atteint 57,5 ans, contre 52 en France). Par ailleurs, une attention particulière a été accordée à la constitution du corpus, afin de varier les situations sociales, tant du point de vue de la catégorie socio-professionnelle des chômeurs que de la durée de chômage. Ils sont répartis équitablement selon le sexe.

Ce mode d'accès au terrain, s'il permet de saisir les effets des politiques de l'emploi et d'analyser leur mise en œuvre, explique que nous avons rencontré des chômeurs plutôt proches de l'emploi, enjoints à en retrouver un. Le risque de réaliser des entretiens sur le chômage en passant par des institutions peut poser plusieurs problèmes, notamment celui de renforcer les discours de « bonne » conduite dans la recherche d'emploi, puisque les discours produits en entretiens sont co-construits selon les conditions d'enquête et en fonction de la manière dont l'enquêté perçoit l'enquêteur (Mauger, 1991). Toutefois, la constitution du corpus s'est accompagnée d'observations propices au recueil de discussions informelles, notamment autour de la recherche d'emploi, sur lesquelles nous revenions en entretien.

Nous proposons de saisir comment le vieillissement actif est réinterprété au sein de chaque espace national, en mêlant niveaux d'analyses micro et macro-sociologiques. Nous développerons notre propos en deux temps. Dans un premier temps, en contextualisant les politiques anglaises et françaises visant les chômeurs âgés ${ }^{3}$, nous verrons que la comparaison des

3. Cette expression, pas très heureuse, témoigne du fait que ce public est désigné par l'âge. 
contextes nationaux fait ressortir la structuration des parcours de vie qu'ils opèrent, ce qui renvoie à la dimension parcours du dossier dans lequel s'inscrit cet article. Par leurs normes et leurs cadres institutionnels, en s'appuyant diversement sur le référentiel du vieillissement actif, ils permettent plus ou moins aux individus d'exprimer le désir de se retirer du marché du travail. Dans un deuxième temps, nous mettrons en exergue que le niveau d'analyse des contextes nationaux ne suffit pas pour comprendre comment les individus se saisissent ou non du vieillissement actif. Il convient également de tenir compte du milieu social, dont les effets se manifestent selon des modalités voisines de part et d'autre de la Manche, ce qui renvoie à la dimension autonomie du dossier de la revue.

\section{Un référentiel modulé par les contextes nationaux}

L’objet ici est de saisir comment se décline le référentiel du vieillissement actif, en France et au Royaume-Uni. Entre-t-il en cohérence avec les politiques publiques de chaque pays ? Pour l'appréhender, nous analysons plus précisément deux dimensions de manière croisée : celle des cadres normatifs et institutionnels nationaux qui structurent l'épreuve du chômage en fin de carrière ; celle du récit que les individus font de leur expérience. La description des cadres permet de contextualiser et d'interpréter les discours des enquêtés ; à l'inverse, les façons de mettre en récit permettent de voir les effets différenciés des cadres institutionnels.

Nous verrons d'abord qu'au Royaume-Uni, le vieillissement actif a une emprise importante. L'expérience du chômage en fin de carrière est associée à la fois à une épreuve financière et morale qui permet peu aux individus d'exprimer le souhait de quitter le marché du travail, d'autant plus que la retraite est dépréciée. En France, le vieillissement actif a une prise limitée en raison d'une culture de la sortie précoce prégnante. De surcroît, l'expérience du chômage en fin de carrière est moins stigmatisée et la retraite est valorisée.

\subsection{Au Royaume-Uni, la prégnance du vieillissement actif}

Au Royaume-Uni, l'expérience du chômage en fin de carrière est associée à une épreuve financière tout autant que morale. L'indemnisation du chômage y est forfaitaire, faible et brève : elle est versée six mois, puis elle passe sous condition de ressources (cf. tableau 1). Selon le principe de less eligibility, les conditions dans le cadre de l'assistance doivent être moins bonnes que celles envisageables dans le cadre d'un emploi salarié (Alcock et al., 2003). 
Tableau 1 : Comparatif des allocations chômage

\begin{tabular}{|c|c|c|}
\hline & $\begin{array}{l}\text { Royaume-Uni : Jobseeker's Allowance } \\
\text { (allocation demandeur d'emploi) }\end{array}$ & France : allocation de retour à l'emploi \\
\hline Montant de l'indemnisation & $\begin{array}{l}\text { Forfaitaire et faible ( } £ 73,10 \text { - environ } 79 € \\
\text { - par semaine pour une personne de plus de } \\
\qquad 25 \text { ans). }\end{array}$ & $\begin{array}{l}\text { Variable selon la durée et le montant des cotisations } \\
\text { (entre } 57 \text { et } 75 \% \text { du salaire de référence) }\left(^{*}\right) \text {. }\end{array}$ \\
\hline Durée d'indemnisation & $\begin{array}{l}\text { Six mois, puis versée sous conditions de } \\
\text { ressources. }\end{array}$ & $\begin{array}{l}\text { Liée à la durée de cotisation ; versée au maximum } 36 \\
\text { mois pour les plus de } 50 \text { ans ( } 24 \text { pour les moins de } 50 \\
\text { ans). }\end{array}$ \\
\hline
\end{tabular}

(*) : En 2014, le montant net moyen mensuel de l'allocation de retour à l'emploi s'élevait à $970 €$.

Source : Auteure.

Au Royaume-Uni, la socialisation du risque chômage est donc limitée et cette dimension est récurrente dans les entretiens. Nombre d'enquêtés se trouvent acculés financièrement. Pour Alan (54 ans, chef de projet $)^{4}$ la situation est "très difficile ». En plus de l'allocation chômage, il perçoit une aide au logement, mais malgré cela, "C'est très dur. J'ai dî emprunter de l'argent ». Le cas de Nick (52 ans, militaire) est similaire : "Si j’ai un entretien d'embauche hors de la ville, j'emprunte la voiture de papa ou il me donne un peu d'argent pour l'essence».

Si les raisons économiques sont avancées comme argument justifiant le retour à l'emploi, elles sont complétées par l'évocation d'un attachement au travail et l'idée plus générale d'être actif. Ces aspects, considérés comme garants de l'autonomie individuelle, sont prégnants au Royaume-Uni (Cole, 2008 ; Riach, Loretto, 2009). L'autonomie y est érigée en norme vivace et elle s'acquiert par l'indépendance financière, qui s'obtient elle-même d'abord par l'emploi. Nick, que nous venons d'évoquer, lie ces raisons économiques et morales : "Je ne veux pas rester assis à la maison et miennuyer, les allocations que je reçois ne sont vraiment pas suffisantes".

Jane (55 ans, enseignante), lorsqu'elle présente sa situation, explique qu'elle doit travailler pour continuer à cotiser et pour rester active : "Quand j'arriverai à l'âge de la retraite, je n'aurai pas assez de cotisations pour percevoir une retraite à taux plein, donc je dois travailler et aussi pour mon propre intérêt, sortir de la maison, j'ai besoin d'avoir quelque chose à faire. "

Ce souhait d'être actif est encore plus saillant quand la situation économique des chômeurs est peu favorable. Le régime anglo-saxon de protection sociale s'enracine dans un modèle normatif valorisant l'activité, qui confère au chômage un caractère moral. L'incitation des individus à travailler est, à tout âge, essentielle. Les politiques de l'emploi sont adossées à un régime libéral d'activation où les individus doivent disposer de ressources minimales garanties par des politiques d'assistance pour affronter la compétition sur le marché. Au-delà, ils doivent assurer eux-mêmes leur protection et ceux qui n’y parviennent pas

4. Dans cette partie, nous indiquons pour chaque chômeur - dont le prénom a été anonymisé - son âge et sa dernière profession. Dans la partie suivante, nous précisons aussi son pays (F pour la France et RU pour le Royaume-Uni). 
sont soupçonnés de profiter de l'assistance. Les débats sur le système de protection sociale s'orientent autour de l'idée de réduire les prestations pour limiter leurs effets potentiellement pervers, en incitant les individus à se prendre en charge et à préserver ainsi leur autonomie (Evans, 2001).

Dans ce contexte, le vieillissement actif se déploie avec force, car il fait écho à ce cadre normatif valorisant l'activité. Il s'y diffuse dès la fin des années 1990, au moment où les travaillistes mettent les fins de carrière à l'agenda. Cette mise à l'agenda n'a été que très peu assortie de dispositifs de politiques de l'emploi spécifiques. D’inspiration libérale en matière de protection sociale, le Royaume-Uni intervient en marge du marché du travail et les politiques de l'emploi sont limitées. La voie politique privilégiée a été de sensibiliser les employeurs via des campagnes de communication et de leur demander d'adopter un code de bonnes pratiques envers les actifs vieillissants. Ce public, tout particulièrement, a peu bénéficié des politiques de l'emploi (Moss, Arrowsmith, 2003) et les dispositifs d'incitation au retour à l'emploi et d'accompagnement spécifiquement tournés vers les chômeurs âgés restent rares. Lancé en 2000, le principal programme à destination de ce public, le New Deal 50 Plus, a eu un impact limité et a été construit sur une base modeste (comparé au New Deal for Young People et au New Deal 25 plus). Non assorti d'objectifs quantifiés, qualifié à ce titre de "petit programme ", il a un reçu un financement modique - 2,1\% des dépenses totales consacrées aux différents New Deal (Hamblin, 2006). À tel point que les intermédiaires de l'emploi rencontrés sur le terrain ne l'évoquaient jamais d'eux-mêmes et que les questions posées à son sujet suscitaient hésitations et incertitudes 5 . Les ateliers de retour à l'emploi où nous avons enquêté sont initiés localement par des prestataires du service public de l'emploi, selon les problématiques du public qui fréquente leur structure.

Si le vieillissement actif fait autant écho dans ce pays - au point où A.-M. Guillemard le qualifie de "culture de l'injonction au vieillissement actif» (op. cit., p. 30) - c'est aussi parce que la retraite, historiquement, y est dévalorisée et envisagée sous l'angle du retrait social et des risques de désocialisation qu'elle implique (Phillipson, 1982). Cette dépréciation de la retraite s'articule à la faiblesse du système des pensions publiques et à la difficulté, pour beaucoup, notamment les femmes et les moins qualifiés, à souscrire à une retraite d'entreprise ou un autre système privé (Ginn, Arber, 1999). Les réformes thatchériennes des années 1980 ont limité le rôle redistributif de l'État, au profit des systèmes privés et ont amoindri les revendications en faveur de l'augmentation des pensions publiques. Les risques de pauvreté et d'exclusion, soulignés par la littérature (Ogg, 2005), sont intériorisés par les individus, d'autant plus que la récession de 2008 a révélé la fragilité des systèmes de retraites privés (Fineman, op. cit.).

5. En outre, l'entrée en vigueur, au moment de l'enquête de terrain, d'un nouveau programme de retour à l'emploi - le Flexible New Deal-, a entraîné la disparition de ce New Deal 50 Plus qui semblait moribond. 
Tableau 2 : Systèmes de retraite anglais et français (régime général)

\begin{tabular}{|l|c|c|c|}
\cline { 2 - 4 } \multicolumn{1}{c|}{} & \multicolumn{1}{c|}{ Âge légal de départ à la retraite } & $\begin{array}{c}\text { Conditions pour la retraite à } \\
\text { taux plein }\end{array}$ & $\begin{array}{c}\text { Taux de } \\
\text { remplacement net }\end{array}$ \\
\hline France & $\begin{array}{c}\text { Depuis 2011, repoussé progressivement de } 60 \text { à } \\
62 \text { ans (selon l'année de naissance) }\end{array}$ & $\begin{array}{c}\text { Condition d'âge (entre } 60 \text { et } 62 \\
\text { ans) et durée minimale d'assurance } \\
(41 \text { ans) } \\
\text { Ou à l'âge du taux plein (entre } 65 \text { et } \\
67 \text { ans selon l'année de naissance) }\end{array}$ & 67,7 \\
\hline Royaume-Uni & $\begin{array}{c}65 \text { ans pour les hommes, } 60 \text { ans pour les femmes } \\
\text { (âge qui recule progressivement pour atteindre } \\
65 \text { ans) }\end{array}$ & $\begin{array}{c}44 \text { ans de cotisation pour les } \\
\text { hommes, 39 pour les femmes }\end{array}$ & 28,5 \\
\hline
\end{tabular}

$\left.{ }^{*}\right)$ : «Le taux de remplacement net se définit comme les droits à retraite individuels nets divisé par le salaire net ». Pour le détail du calcul de ce taux, nous renvoyons à $O C D E, 2016$.

Source : OCDE, 2016.

La plupart des chômeurs estiment ainsi que leur pension sera insuffisante. Ils se montrent fatalistes par rapport aux réformes et dénigrent ce temps de la vie. Là encore, raisons économiques et morales se mêlent pour justifier l'idée de repousser la retraite et continuer à travailler. Ces éléments permettent de comprendre pourquoi ce temps social fait l'objet de peu d'aspirations, beaucoup moins qu'en France. Même lorsqu'une aspiration à la retraite est formulée, elle est souvent nuancée. La retraite tient, par exemple, une place importante dans le récit de Jane (55 ans, enseignante), qui la considère comme un acquis social. Pourtant, elle pondère son désir d'être à la retraite : "Si je pouvais prendre ma retraite à taux plein, je la prendrais demain! [elle rit] Non c'est idiot. Ce n'est pas vrai, parce que travailler, même à mi-temps, c'est très bien et je pense que c'est essentiel pour rester socialisé. [...] Je n'aimerais pas rester à la maison toute la journée, juste regarder la télévision, parce que je crois que je deviendrais folle."

Dans son discours, le temps de la retraite renvoie au repli sur soi et à un niveau de vie dégradé. Réaffirmer son attachement au travail permet aussi de se démarquer de l'image négative de l'inactivité. Plus généralement, quasiment tous les chômeurs anglais ont revendiqué le droit de travailler à la retraite, ce qui témoigne des difficultés financières qu'ils sont nombreux à anticiper (une caractéristique des régimes libéraux de protection sociale ; Lesemann, Beausoleil, 2004), mais aussi de la prégnance du vieillissement actif dans les projections des individus vis-à-vis de la retraite ; en effet, tandis que ce référentiel valorise le travail, il délégitime le droit au repos (Macnicol, op. cit.).

Ainsi, au Royaume-Uni, le vieillissement actif structure l'expérience du chômage en fin de carrière. Il s'articule à un cadre institutionnel et normatif qui valorise l'activité à tout âge, qui déprécie les périodes de non-travail dont les individus cherchent à se tenir à distance. Dans ce contexte, il est difficile d'aspirer à la retraite. Pour la minorité qui formule cette aspiration, elle est contrebalancée par le fait de vouloir rester actif. Cette norme se retrouve dans les récits de tous les chômeurs anglais, même si son intensité varie, comme nous le verrons, selon le milieu social. L'attachement des chômeurs anglais âgés au travail paraît donc fort. Ce résultat semble contradictoire avec les enquêtes européennes sur le rapport 
au travail qui révèlent que le travail est jugé peu important par les britanniques (Méda, Vendramin, 2013) - et même moins important qu'il ne l'est pour les français. Le rapport au travail est complexe, mais de nombreuses recherches ont établi que le statut d'emploi y est déterminant. Pour les chômeurs, le travail est plus souvent considéré comme très important (Baudelot, Gollac, 2003 ; Méda, Vendramin, Ibid.), ce qui explique ce décalage avec les enquêtes quantitatives.

\subsection{En France, une emprise moins forte du vieillissement actif}

Dans les entretiens français, l'expérience du chômage est, comme au Royaume-Uni, une épreuve marquée par l'incertitude. La plupart des enquêtés sont inquiets quant à l'issue de cette situation. Ils appréhendent une baisse de leur niveau de vie en cas de chômage prolongé. L'absence d'emploi est stigmatisante, mais elle semble moins mal vécue.

Florence affirme ainsi : "C'est pas parce que je trouve pas de boulot que je suis nulle quoi." (50 ans, prestataire culturelle).

Jean-Paul, pour sa part, assure : "Je peux encore être utile sur le marché du travail, maintenant si je trouve pas, je vais pas me suicider! Je vivrai modestement et puis j'attendrai l'heure du départ. Que je saurai occuper assez bien d'ailleurs. " (57 ans, éducateur spécialisé).

L'inactivité fait l'objet de moins d'appréhension et de stigmatisation. De plus, les individus sont moins soumis à l'épreuve temporelle du chômage. Du moins, l'urgence financière surgit plus tardivement et l'inquiétude est moins palpable dans les entretiens. Le système d'indemnisation du chômage permet en effet aux individus de se sentir moins vite acculés financièrement. Le témoignage de Jean-Paul montre ainsi qu'il n'est pas inquiet. À propos de l'allocation, il précise : "C'est deux ans je crois ou deux ans et demi, je sais plus, je me suis même pas renseigné, je m'en fous, c'est pas que j'ai pas besoin d'argent, mais ça me préoccupe pas" (57 ans, éducateur spécialisé). Florence, bientôt en fin de droits, assure : "J'ai appris à vivre avec ce que j'avais " (50 ans, prestataire culturelle).

Par ailleurs, plusieurs chômeurs justifient leurs démarches vers l'emploi uniquement par des raisons financières. Ainsi, Patrice déclare : "Il faut que je me ressaisisse pour regagner des sous" (52 ans, DRH). Louise affirme, pour sa part, que le chômage, "c'est surtout au niveau financier que ça [l]'emmerde" (51 ans, comptable). De son côté, Nadège explique ne pas avoir cherché tout de suite. L'anticipation de la baisse prochaine de son allocation a fonctionné comme un déclic: "Quand j'ai vu l'échéance arriver, mi-novembre, ding ding ding ding ding! [rires] Ah! Je me suis dit non, mais attends, il faut que je me bouge là.»(47 ans, responsable des ventes).

Les arguments qui incitent à rechercher un emploi ne sont donc pas les mêmes de part et d'autre de la Manche : au Royaume-Uni, ils sont à la fois financiers et moraux, mais les premiers sont rarement énoncés seuls ; alors qu'en France, ils peuvent être évoqués comme raison principale. 
Comme au Royaume-Uni, le système d'indemnisation du chômage est lié à un cadre normatif spécifique. En France, celui-ci est davantage gouverné par un principe de solidarité qui s'accompagne d'une épreuve du chômage moins stigmatisante, moins réduite à la responsabilité individuelle (Demazière et al., 2013). De plus, le risque chômage est davantage socialisé (cf. supra) et les chômeurs de plus de 50 ans bénéficient d'une indemnisation plus longue. Cette mesure est justifiée par les difficultés d'accès à l'emploi de ce groupe d'âge. Elle témoigne en outre d'une culture de la sortie précoce toujours prégnante (Guillemard, op. cit.).

Si la France a mis à l'agenda l'emploi des seniors au cours des années 2000, les mesures d'incitation au retour à l'emploi et d'accompagnement des chômeurs âgés dans leur recherche d'emploi restent en effet limitées. En 2006, un CDD (contrat à durée déterminée) senior a été introduit dans le Code du travail, mais il est peu utilisé par les entreprises. À partir de 2008, le service public de l'emploi a été mobilisé et des ateliers de recherche d'emploi ont été mis en œuvre pour ce public de manière locale, non systématique. Le nombre de contrats aidés a augmenté pour les plus de 50 ans et ceux-ci peuvent en bénéficier plus longtemps (36 mois au lieu de 24, voire jusqu'à la retraite pour les chômeurs de plus de 58 ans). En outre, les conditions d'accès à la retraite ont été durcies (même si la réforme de 2014 atténue les inégalités, notamment en matière de pénibilité) et les règles du cumul emploi-retraite ont été assouplies.

Pour les pouvoirs publics, l'enjeu a surtout été de contenir les sorties anticipées du marché du travail en supprimant les dispositifs existants (préretraites, dispense de recherche d'emploi), mais dans le même temps, d'autres dispositions sont apparues, permettant les départs anticipés (pour carrière longue, pour pénibilité). Dans ce contexte où coexistent des dispositifs invitant à la prolongation des carrières et d'autres permettant les sorties précoces du marché du travail, parler des chômeurs âgés en termes d'activation des politiques de l'emploi n'a rien d'évident.

En conséquence, le référentiel du vieillissement actif a trouvé moins d'écho en France et il transparaît moins dans les récits des chômeurs. Malgré les réformes des retraites et l'assouplissement des conditions de cumul emploi-retraite, travailler après avoir liquidé ses droits demeure minoritaire (Govillot, 2013). Laspiration à la retraite est forte, la retraite étant un temps désiré et valorisé. Les individus sont nombreux à se positionner contre les réformes et beaucoup aspirent à partir le plus tôt possible (même si, nous le verrons, les positions varient socialement). Les chômeurs parlent aisément des projets qu'ils font pour ce temps de la vie et le mettent moins à distance. Guy précise, par exemple : "J'y pense, préparer ma retraite, c'est maintenant" (55 ans, responsable de production). Les individus semblent moins inquiets par rapport à la retraite, rares sont ceux qui déclarent que leur pension sera insuffisante.

L'attachement au travail est plus ambivalent (Méda, Vendramin, op. cit.) : tout en le considérant comme important, ils sont nombreux à souhaiter qu'il occupe moins de place dans leur vie. Des facteurs liés à l'intensification du travail et à la pénibilité permettent d'expli- 
quer ce détachement par rapport au travail en fin de carrière (Molinié, 2005), d'autant que les sorties anticipées du marché du travail restent légitimées par des dispositifs les autorisant. Dans ce contexte, le référentiel du vieillissement actif se décèle peu, ce dont témoignent les aspirations à la retraite, socialement admises.

Ainsi, le vieillissement actif trouve des échos distincts selon les contextes nationaux. Le désir de se retirer du marché du travail est plus ou moins vif dans la mesure où les édifices normatifs et institutionnels légitiment ou non de se détacher du travail en fin de carrière. Au Royaume-Uni, les périodes de non-travail sont dévalorisées et peu indemnisées. En France, les normes et les mesures rendent l'expérience du chômage en fin de carrière moins pénible.

Les précédents constats permettent de saisir la cohérence de chaque contexte national. Chacun définit un cadre normatif qui s'articule à un système de protection sociale. Cadres normatifs et institutionnels structurent les parcours de fin de carrière. Néanmoins, ces constats risquent de masquer des clivages sociaux et de donner une vision homogénéisante des parcours et des manières d'envisager sa place sur le marché du travail. Dans la seconde partie, nous affinons les analyses en montrant que les espaces nationaux sont traversés par des contrastes sociaux qui s’articulent aux spécificités nationales.

\section{Une réception socialement différenciée du « vieillissement actif »}

Partant du constat que le rapport au chômage se décline différemment selon les milieux sociaux (Schnapper, 1994 ; Parry, Taylor, 2007), nous avons analysé la manière dont les chômeurs âgés évoquent leur situation ainsi que leur rapport à la retraite. Examiner la manière dont les individus envisagent leur place sur le marché du travail en fin de carrière permet de montrer que, dans chaque pays, la sensibilité au vieillissement actif varie socialement. Nous avons identifié quatre manières de se positionner. Cette typologie est fondée sur différentes variables (catégorie socio-professionnelle, durée de chômage et âge). Nous verrons que dans le premier cas, les individus sont sensibles au vieillissement actif et, avec les difficultés à retrouver un emploi, ils intériorisent les préconisations de ce référentiel (deuxième cas). Dans le troisième cas, les individus sont dans une situation qui les contraint à rester «actifs » sur le marché du travail, quelles que soient leurs aspirations. Enfin, le quatrième type réunit les expériences d'individus qui aspirent à la retraite et qui s'opposent aux préconisations qui découlent du vieillissement actif.

\subsection{Sensibilité au vieillissement actif}

Dans le premier cas (minoritaire), les individus sont sensibles au vieillissement actif. Ces enquêtés partagent plusieurs traits : ils sont diplômés et ont jusqu'à présent connu une carrière valorisante et ascendante qui les a amenés à occuper des positions hiérarchiques 
élevées, de cadre supérieur ou de manager. Ce sont également fréquemment des hommes. Depuis peu au chômage, ils disposent de ressources financières qui les mettent pour un temps à l'abri du besoin.

En France, ils bénéficient d'une allocation chômage confortable, souvent associée à une prime de départ. Au Royaume-Uni, ils comptent moins sur l'allocation que sur leurs économies et des primes de licenciement. Ce qui leur permet d'échapper, souvent, au service public de l'emploi anglais envers lequel ils se montrent critiques, ce qui leur évite de se sentir stigmatisés (Warin, 2011). En outre, ils ont le sentiment d'avoir une valeur professionnelle. Ils estiment avoir des compétences rares et prisées, une expérience intéressante qui les rassure quant à leur retour à l'emploi, qu'ils prévoient rapide et dans des conditions avantageuses. Dans ce contexte, ils investissent la recherche d'emploi comme un métier.

De part et d'autre de la Manche, les individus perçoivent la retraite sur un mode lointain : elle constitue une perspective à laquelle ils aspirent, mais qu'ils envisagent de repousser à des fins d'épanouissement personnel, selon leurs opportunités. Ce rapport à la retraite est caractéristique des individus qui occupent les positions professionnelles les plus élevées (Parry, Taylor, op. cit.; Aouici et al., 2008). Guy affirme : "Je me vois pas encore à la retraite» (F, 55 ans, responsable de production). Esther (F, 50 ans, journaliste), aimerait quant à elle "travailler jusqu'à 60 ans et au-delà ".

Pour eux, l'emploi post-carrière ne s'envisage pas tellement pour des raisons financières car, davantage que les autres chômeurs, surtout anglais, ils ont pu souscrire à des retraites d'entreprise ou des fonds de pension qui n'ont pas été mis à mal lors de la récession de 2008. Chris aimerait, pour sa part, continuer à travailler « quelques jours par semaine ou un jour par semaine aussi longtemps que je le pourrai " (RU, 60 ans, ingénieur). Le sentiment d'avoir le choix, l'âge venu, de prendre ou reporter la retraite n'est pas courant dans le corpus anglais. Ian a une pension confortable en vue ; il bénéficiera d'une retraite d'entreprise, d'un fond de pension et de la retraite d'État : "Je me sens prêt à travailler encore, je sais pas... vous voyez, certains veulent prendre leur retraite à 60 ans ou le plus tôt possible et ne plus travailler. J'aime l'idée de retraite, j'ai beaucoup de choses à faire quand je prendrai ma retraite et j'ai fait beaucoup de choses lorsque je n'avais pas de travail, mais je suis content de travailler aussi. " (RU, 60 ans, ingénieur)

En outre, ces enquêtés anglais dénigrent moins la retraite, par rapport à leurs compatriotes. Si l'attachement au travail est présent dans leur récit, la retraite comme période d'inactivité n'est pas crainte. Les individus identifient l'âge légal de la retraite et ils imaginent le repousser. Ce n'est pas pour autant qu'ils tiennent des discours sur la nécessité de réformer les retraites. Au Royaume-Uni, les réformes suscitent en fait peu d'intérêt pour ces individus, qui ne se sentent guère concernés. Les propos de Ian (RU, 60 ans, ingénieur) sont éloquents. Il sait que la réforme de 2007 qui augmente l'âge de départ ne l'affectera pas, "ce sera toujours 65 ans pour moi ». Au-delà, avoue-t-il, il y a peu réfléchi, comme en témoignent ses nombreuses hésitations : "Euh... je ne [il se reprend], je ne pense pas que ça soit nécessairement une bonne chose, mais euh... vous voyez, je n'y ai pas vraiment 
beaucoup pensé. Euh... mais... j’aime l'idée que peut-être, si à 65 ans vous êtes content de faire ce que vous faites et que l'entreprise accepte de vous garder, alors.. c'est bien de pouvoir continuer. Oui. Je... je ne sais pas, je ne suis pas particulièrement inquiet par rapport à tout ça. J'ai aucune... j'ai pas vraiment d'opinion là-dessus. »

En France, en revanche, la réforme de 2010 provoque des réactions. Guy (F, 55 ans, responsable de production) s'y oppose. Pour lui, il faut tenir compte des carrières longues. Esther (F, 50 ans, journaliste) s'insurge contre le fait que la pénibilité du travail n'ait pas été prise en compte : "Personnellement ça me gêne pas trop [...], mais pour ceux qui ont travaillé dur c'est dégueulasse. "

\subsection{Intériorisation du vieillissement actif}

Dans le deuxième idéal-type, les individus adhèrent davantage au vieillissement actif. Comme dans le cas précédent, cette position est minoritaire et concerne des chômeurs ayant occupé des fonctions de cadre supérieur ou de manager. Ils sont bien dotés en titres scolaires, ont le sentiment de détenir des compétences rares sur le marché du travail et ont eu des responsabilités professionnelles. Ce type réunit également des expériences plutôt masculines.

La principale différence réside dans l'ancienneté au chômage : souvent depuis plus d'un an au chômage, ces individus font l'expérience de difficultés dans l'accès à l'emploi. Certains voient leur situation se dégrader et éprouvent une insécurité statutaire. S’ils se sentent en difficulté, ils restent néanmoins sûrs de leur valeur professionnelle et ils considèrent que l'âge est l'obstacle principal à leur retour à l'emploi. Ils l'associent à un carcan, conférant au chômage un caractère irréversible et injuste. Les récits sont alors organisés autour de la dénonciation de la discrimination sur l'âge. Les individus cherchent à montrer son caractère illégitime, afin de réaffirmer la légitimité de leur place sur le marché du travail. Du point de vue de la retraite, deux éléments les caractérisent.

D'une part, pour justifier leur place sur le marché, ils mettent à distance ce temps de la vie. Ils envisagent de prendre leur retraite le plus tard possible. Charles (F, 59 ans, cadre technique), par exemple, retrace un échange avec une conseillère à l'emploi qui lui demande s'il s'est fixé une échéance. Dans la réponse qu'il raconte lui avoir faite, il pense repousser son départ après l'âge canonique de 60 ans : "La loi française prévoit quion peut travailler jusqu’à 65. Et même au-delà dans certains cas, administrateurs, etc. Où est le problème? Moi je veux travailler jusqu'à 65 ans."

Martin (RU, plus de 65 ans, manager) estime, pour sa part, qu'il ne prendra sa retraite que lorsqu'il ne pourra plus travailler et non en fonction de son âge. Il n’aspire pas à ce statut, dont il a une vision négative

D'autre part, ces personnes se montrent favorables aux réformes qui visent à allonger les carrières professionnelles. Elles reprennent à leur compte les arguments économiques et 
démographiques pour les légitimer. Xavier, par exemple, s'exclame : "Je suis effaré devant la connerie et la manipulation des gens, parce que c'est clair qu'avec, d'une part, l'allongement de la durée de la vie, et le fait que le nombre de retraités augmente massivement par rapport au nombre d'actifs, il est évident qu'y a pas assez d'argent pour payer tout le monde, donc pour moi c'est évident, ça coule de source. D'ailleurs, le gouvernement, qu'est-ce qu'ily peut? Il y peut rien!» (F, 51 ans, commercial)

De leur point de vue, la retraite ne doit pas être imposée. Thierry (F, 52 ans, responsable commercial) s'indigne: "Mais pourquoi interdire aux gens de travailler après 60 ans ?! De quel droit? " De la même manière, Martin estime : "Les gens devraient avoir le choix, sils veulent prendre leur retraite ou non, et sils choisissent de continuer à travailler, ils devraient être autorisés à continuer, c'est aussi simple que ça. " (RU, plus de 65 ans, manager). Agnès (F, 50 ans, commerciale) partage cette vision : "Je connais autour de moi beaucoup de gens qui à 50 ans ont envie de travailler encore 10 ans quoi. Et d'autres qui sont très malheureux de partir».

Se démarquer de la retraite, approuver les réformes leur permet de réaffirmer la légitimité de leur place sur le marché du travail. En cela, ils sont proches des préconisations du vieillissement actif et des orientations politiques sur l'allongement de la vie professionnelle.

\subsection{Nécessité économique du vieillissement actif}

Dans cette troisième position, qui représente l'expérience majoritaire parmi les chômeurs rencontrés, en particulier au Royaume-Uni et pour les femmes, la retraite constitue une perspective lointaine qui va de pair avec la nécessité économique du retour à l'emploi. Plus souvent célibataires et/ou ayant encore des enfants à charge, ils sont éloignés de l'âge légal de la retraite. En outre, ils sont moins diplômés : on retrouve des professions intermédiaires, des employés, des cadres et des managers autodidactes.

Leur carrière est marquée par des ruptures et des expériences antérieures du chômage. Pour eux, le retour à l'emploi constitue une nécessité qui leur semble pourtant délicate à réaliser : ils identifient l'âge comme frein indépassable.

Pour eux, la retraite constitue un horizon lointain. En France, l'aspiration à la retraite est forte, même si cette perspective reste éloignée en raison de l'âge des individus. Elle représente un statut moins stigmatisant et plus rémunérateur que le chômage : "Dans neuf ans, je serai tranquille ", déclare Thierry (F, 52 ans, responsable commercial).

Les mesures d'âge ne sont guère discutées, même s'ils regrettent l'utilisation de ce critère dans les opérations de recrutement qui entrave leur accès à l'emploi. Ils s'opposent en majorité au report de l'âge légal de départ en retraite. Florence, par exemple, a participé aux manifestations qui ont eu lieu en 2010 au moment où la loi sur les retraites était débattue : "Je trouve tellement ridicule de demander aux gens de travailler plus longtemps, alors qu'à 45 ans, il nous est difficile de trouver du boulot. » (F, 50 ans, prestataire culturelle) 
Au Royaume-Uni, en revanche, les avis sont partagés. Certains sont défavorables aux réformes qui visent à augmenter les durées de vie au travail. Ray (RU, 57 ans, manager) estime que, dans une conjoncture difficile, elles auront pour effet d'accroître le chômage des plus âgés : "Si vous avez un marché morose, vous allez avoir trop de chômeurs, alors vous n'allez pas employer les plus âgés, parce qu'ils ont moins d'années à offrir et aussi moins de flexibilité".

Pour lui, ces réformes permettent au gouvernement de faire des économies car "payer des gens au chômage avec l'allocation chômage revient moins cher que payer une retraite". Cette position semble pourtant minoritaire. Dans le corpus, ils sont nombreux à défendre le droit au travail à tout âge dans la mesure où ils anticipent l'obligation qu'ils auront de continuer à travailler lorsqu'ils atteindront l'âge de la retraite. Pour eux, l'emploi post-carrière apparaît comme une nécessité (Lesemann, Beausoleil, 2004). D'autres encore se montrent peu informés et relativement indifférents, ayant intériorisé la faiblesse probable de leurs revenus dans ce temps de la vie éloigné. Compte tenu de ses difficultés à retrouver un emploi, Nick (RU, 52 ans, militaire) aspire à la retraite, même s'il n'a pas d'idée précise du montant de sa pension : "Je n'en ai aucune idée. Non, ce ne sera pas beaucoup."

\subsection{Défense du droit au repos}

Une quatrième position par rapport au vieillissement actif émerge des entretiens, celle de la défense d'un droit au repos via la défense de la retraite. Ces chômeurs affirment aspirer à partir le plus tôt possible. Cette position, minoritaire dans le corpus, est plutôt tenue par des femmes, proches de l'âge légal de la retraite, de milieux sociaux souvent modestes. Ils ont occupé des fonctions peu qualifiées, d'ouvriers ou d'employés, ou ont connu une rupture dans leur parcours professionnel qui les a amenés à se reconvertir dans le secteur social. Ces enquêtés se sentent peu au chômage et la recherche d'emploi est faiblement investie. Certains, en raison de leur état de santé, ne souhaitent pas retrouver d'emploi. Ils mettent à distance le travail et aspirent à d'autres activités, même si les préretraites leur sont aujourd'hui moins accessibles, ce qui implique de devoir chercher un emploi ou de "faire comme si " (Monique, F, 58 ans, secrétaire).

En outre, cette mise à distance du travail s'articule au sentiment d'avoir "passé l'âge " et d'avoir "suffisamment travaillé ". Ils se sentent vieillir, ce qui se traduit par deux préoccupations qui leur permettent de renforcer leur mise à distance du travail : celle du temps qu'il leur reste à vivre et celle de leur santé à préserver. Tarun (RU, 61 ans, commerçant) confie : "Quand vous atteignez 50-60 ans, c'est important de préserver sa santé ». Hilary (RU, 56 ans, employée) déclare ainsi que l'argent ne fait "pas tout» et que : "le temps, c'est quelque chose que vous ne récupérez jamais et aujourd'hui je sens que... vous savez, j'ai souvent travaillé 10, 12 heures par jour, parfois même plus, sept jours dans la semaine parfois et je pense qu'aujourd'hui je voudrais juste améliorer ma pension 
pour que j'aie assez d'argent pour vivre et avoir un peu de temps pour les choses que je veux. Quand vous êtes mort, comme ils disent, vous avez beaucoup de temps [elle rit]. Mon frère me dit toujours "ne sois pas la personne la plus riche du cimetière"!"

En outre, ces chômeurs se montrent attachés à la retraite et à l'âge légal de départ. Ils ne font preuve d'aucune ambivalence par rapport aux réformes en cours, ils s'y opposent. D'ailleurs, plus que les autres chômeurs, ils sont au courant des réformes, ils se sont intéressés aux conséquences qu'elles auraient pour eux. L'entretien réalisé avec Jean-Paul s'est tenu au moment de la réforme des retraites de 2010, en France. Pour lui, celle-ci ne se justifie pas et il met en cause les arguments habituellement énoncés pour la légitimer : "Faire travailler les gens... on sait très bien qu'il y a de l'argent hein, mais comme ils veulent pas le lâcher, qui est-ce qui va payer? Ben c'est toujours ceux qui bossent. [...] ce que je crains c'est que derrière tout ça, y'ait des projets de tuer la retraite par répartition au profit d'une retraite par capitalisation. "(F, 57 ans, éducateur spécialisé)

Par ailleurs, le temps de la retraite n'est pas non plus dénigré par les chômeurs anglais, ce qui est rare dans le corpus. Ils envisagent la retraite comme un droit. Hilary (RU, 56 ans, employée) prend position par rapport à ses concitoyens qui l'envisagent comme une cause de désocialisation et de dégradation de la santé : "J'en ai bien envie, j'ai travaillé toute ma vie, je suis impatiente d'y être! [elle rit] Je ne vois aucune raison d'avoir peur. Non. Je connais des personnes qui pensent qu'elles ne vont pas réussir à gérer si elles n’ont rien. Mais si vous le pensez, il y a plein de bénévolat à faire. "

La retraite constitue pour elle un droit "mérité», "gagné». Quant à Jane (RU, 55 ans, enseignante), elle sait qu'elle pourra partir à 64 ans et 10 mois et elle se positionne contre les réformes qui allongent les carrières, estimant qu'elles sont absurdes et qu'elles les placent dans des situations intenables : " $\grave{A}$ moins que les entreprises commencent $\grave{a}$ tenir compte du fait qu'il va y avoir plus de vieux, il n'y aura pas de travail pour eux et quel type d'emploi ils s'attendent à ce que ces personnes puissent faire? On peut pas tous travailler dans des supermarchés à remplir des étagères!"

\section{Conclusion}

Le vieillissement actif vise à inciter les individus à rester actifs et autonomes le plus longtemps possible. Nous avons montré que ce référentiel ${ }^{6}$ se diffuse de manière différenciée dans les contextes français et britannique. L'articulation de deux niveaux d'analyse met en évidence que les expériences du chômage en fin de carrière sont structurées à la fois par les cadres nationaux - les normes et les règles institutionnelles en vigueur - et par des rapports sociaux.

6. On rappelle que la notion de référentiel renvoie à des normes et des valeurs qui produisent des cadres d'interprétation, notamment aux programmes d'action publique. 
Le croisement de ces niveaux d'analyse permet, d'une part, de saisir les réactions individuelles face aux politiques publiques visant à allonger les carrières professionnelles et, d'autre part, de rendre compte de la manière dont les parcours sont socialement construits.

Dans le contexte anglais, l'urgence financière plus forte va de pair avec l'expression d'un attachement au travail et amène à présenter l'inactivité comme intolérable et ce, d'autant plus dans un contexte de dévalorisation de la retraite. En France, le système de protection sociale adossé à un édifice normatif marqué par la culture de la sortie précoce, amortit cette épreuve. Il permet d'aspirer légitimement à la retraite et de l'énoncer en entretien. Cette mise en perspective permet de nuancer l'idée d'un acteur rationnel (Dubet, Vérétout, 2001) qui procéderait à un calcul des coûts et des avantages pour envisager, par exemple, un retrait plus ou moins précoce du marché du travail. Les comportements s'éclairent certes par leur mise en perspective avec les prestations auxquelles les individus peuvent accéder, mais ils se saisissent surtout au regard de normes qui légitiment certains comportements d'emploi et en délégitiment d'autres.

Par ailleurs, dans chaque pays, on retrouve des positions socialement distinctes par rapport au vieillissement actif. Elles traduisent des rapports différenciés à l'emploi en fin de carrière. Elles mettent en exergue que la sensibilité aux normes et aux valeurs sous-jacentes au vieillissement actif émane, de part et d'autre de la Manche, de cadres supérieurs et de managers. De surcroît, ce sont plus souvent des hommes en bonne santé, dotés de diplômes ou de compétences qui leur permettent d'être encore compétitifs sur le marché du travail qui ont connu une carrière professionnelle ascendante et valorisante. Ce sont eux qui, les premiers, encouragent les réformes des retraites et rejettent toutes mesures d'âge qui constituent pour eux un carcan, alors que pour beaucoup d'autres, elles permettent d'échapper à un marché du travail où la pénibilité n'a pas disparu (Gollac, Volkoff, 2006 ; Robinson, Smallman, 2006).

\section{Bibliographie}

Alcock P., Beatty C., Fothergill S., Macmillan R., Yeandle S. (2003), Work to Welfare, Cambridge: Cambridge University Press.

Aouici S., Carillon S., Mette C. (2008), « Choisir l'âge de sa retraite : un arbitrage lié aux contextes économiques et professionnels ", Retraite et société, n 54, pp. 190-209.

Baudelot C., Gollac M. (dir.) (2003), Travailler pour être heureux?, Paris, Fayard.

Barbier J.-C. (2002), " Peut-on parler d'activation de la protection sociale en Europe ?", Revue française de sociologie, $\mathrm{n}^{\circ}$ 43-2, pp. 397-332.

Biggs S. (2001), "Toward critical narativity: stories of aging in contemporary social policy", Journal of Aging Studies, n 15, pp. 303-316. 
Cole M. (2008), "Sociology contra governement? The contest for the meaning of unemployment in UK policy debates", Work, Employment and Society, n ${ }^{\circ}$ 1, pp. 27-43.

Demazière D., Guimarães N. A., Hirata H., Sugita K. (2013), Etre chômeur à Paris, São Paulo, Tokyo, Paris, Presses de Sciences Po.

Dubet F., Vérétout A. (2001), «Une “réduction” de la rationalité de l'acteur. Pourquoi sortir du RMI ?", Revue française de sociologie, n 42-3, pp. 407-436.

Duncan G. (2008), “The dangers and limitations of equality agendas as means for tackling old-age prejudice”, Ageing \& Society, n 28, pp. 1133-1158.

Evans M. (2001), "Britain: moving towards a work and opportunity-focused welfare state”, International Journal of Social Welfare, $\mathrm{n}^{\circ}$ 10, pp. 260-266.

Fineman S. (2011), Organizing Age, Oxford: Oxford University Press.

Ginn J., Arber S. (1999), "Changing patterns of pension inequality: the shift from state to private sources”, Ageing \& Society, n 3, pp. 319-342.

Gollac M., Volkoff S. (2006), "La santé au travail et ses masques », Actes de la recherche en sciences sociales, $\mathrm{n}^{\circ} 163$, pp. 4-17.

Govillot S. (2013), "Le passage de l'emploi à la retraite ", Insee Première, n 1449.

Guillemard A.-M. (2010), Les défis du vieillissement. Âge, emploi, retraite. Perspectives internationales, Paris, Armand Colin.

Guilllemard A.-M. (2013), « Le vieillissement actif : enjeux, obstacles, limites. Une perspective internationale ", Retraite et société, $\mathrm{n}^{\circ}$ 65, pp. 17-38.

Hamblin K. (2006), Older and Unemployed: The experience of unemployment and the new configuration of 'rights and responsabilities' in the $U K$, Bath, European Research Institute, Working Paper Series n ${ }^{\circ} 6$.

Jolivet A. (2002), "La politique européenne en faveur du vieillissement actif ", Retraite et société, $\mathrm{n}^{\circ} 36$, pp. 137-157.

Lesemann F., Beausoleil J. (2004), "Les emplois “post-carrière” aux États-Unis ", Retraite et société, $\mathrm{n}^{\circ} 42$, pp. 9-43.

Macnicol J. (2006), Age Discrimination. An Historical and Contemporary Analysis? Cambridge: Cambridge University Press.

Mauger G. (1991), "Enquêter en milieu populaire », Genèses, n 6, pp. 25-143.

Méda D., Vendramin P. (2013), Réinventer le travail, Paris, PUF.

Molinié A.-F. (2005), "Se sentir capable de rester dans son emploi jusqu’à la retraite ? ", Pistes, $\mathrm{n}^{\circ} 7-1$. 
Moss N., Arrowsmith J. (2003), A review of 'what works' for clients aged over 50, Sheffield, DWP.

Muller P. (2010), "Référentiel », in Boussaguet L. et al., Dictionnaire des politiques publiques, Paris, Presses de Sciences Po, pp. 555-562.

Ogg J. (2005), "Social exclusion and insecurity among older Europeans: the influence of welfare regimes", Ageind \& Society, n 1, pp. 69-90.

OCDE (2016), Panorama des pensions 2015, Éditions OCDE, Paris.

Parry J., Taylor R. F. (2007), “Orientation, opportunity and autonomy: why people work after state pension age”, Ageing \& Society, n² 27, pp. 579-598.

Phillipson C. (1982), Capitalism and the Construction of Old Age? London: Macmillan Press.

Riach K., Loretto W. (2009), "Identity work and the 'unemployed' worker: age, disability and the lived experience of the older unemployed", Work, employment and society, $\mathrm{n}^{\circ} 1$, pp. 102-119.

Robinson A. M., Smallman C. (2006), “The contemporary British workplace: a safer and healthier place?”, Work, employment and society, vol. 20, n 1, pp. 87-107.

Schnapper D. (1994), L'épreuve du chômage, Paris, Gallimard.

Viriot Durandal J.-P., Moulaert T. (2014), «Construction et diffusion d'un nouveau référentiel d'action publique international en faveur d'une politique de vieillissement : le cas du vieillissement actif ", Socio-Logos, $n^{\circ} 9$.

Warin P. (2011), "Le non-recours par désaccord ", in Jaeger M., Usagers ou citoyens : De l'usage des catégories en action sociale et médico-sociale, Paris, Dunod, pp. 115-136. 